SECTION 25. Technologies of materials for the light and textile industry.

Bekzhan Abzalbekov doctoral student of the Department «Technology of textile industry» Taraz State University named after M.Kh. Dulati, Kazakhstan

Alexandr Nikolayevich Shevtsov candidate of technical sciences, corresponding member of the Kazakhstan National Academy of Natural Sciences, President of International Academy of T\&AS (USA, Sweden, Kazakhstan), Department of «Mathematics», Deputy Director on Science of faculty of information technologies, automation and telecommunications, Taraz State University named after M.Kh. Dulati, Kazakhstan Shev_AlexXXXX@mail.ru

\title{
THE CALCULATION OF THE OPTIMAL COEFFICIENTS FOR NORMAL DISTRIBUTIONS
}

\begin{abstract}
In this article, on the basis of statistical data of anthropometric studies shrub diabetes is the calculation of the optimal coefficients for normal distributions, the closest to normal distribution.

Key words: normal distribution, statistical data analysis, diabetes research.

Citation: Abzalbekov B, Shevtsov AN (2014) THE CALCULATION OF THE OPTIMAL COEFFICIENTS FOR NORMAL DISTRIBUTIONS. ISJ Theoretical \& Applied Science 9 (17): 132-140. doi: http://dx.doi.org/10.15863/TAS.2014.09.17.23
\end{abstract}

\section{РАСЧЕТ ОПТИМАЛЬНЫХ КОЭФФИЦИЕНТОВ ДЛЯ НОРМАЛЬНЫХ РАСПРЕДЕЛЕНИЙ}

\begin{abstract}
Аннотация: $B$ данной статье на основе статистических данных антропометрических исследований стом больных сахарным диабетом делается расчет оптимальных коэффициентов для нормальных распределений, наиболее приближенным к нормальному распределению.
\end{abstract}

Ключевые слова: нормальное распределение, статистические данные, анализ, диабет, исследование.

\section{ВВЕДЕНИЕ}

Антропометрическое исследования стопы больных диабетом проведем по методике описанной в [1, c.113]. Данные обмера обрабатываем методами математической статистики. Для каждого из размерных признаков определяем такие статистические параметры, которые характеризуют величину и вариабельность признака в выборке. Как бы ни была однородна изучаемая группа людей, значения любого из антропометрических признаков подверждены изменчивости, различные значения любого из антропометрических признаков встречаются с разной частотой - одни чаще, другие реже. Для характеристики вариабельности антропометрических признаков составили вариационные ряды размерных признаков, которые представлены в таблице 1. Он представляет собой двойной ряд чисел, состоящий из значений признака, сгруппированных в классы, и соответствующих каждому классу частот (вариантов). 
Таблица 1

\section{Вариационные ряды по размерным признакам}

\begin{tabular}{|c|c|c|c|c|c|c|c|c|}
\hline \multirow{2}{*}{$№$} & \multicolumn{2}{|c|}{$\begin{array}{c}\text { Вариационный ряд } \\
\text { по Дс }\end{array}$} & \multicolumn{2}{c|}{$\begin{array}{c}\text { Вариационный ряд по } \\
\text { Ш }_{\text {нп }}\end{array}$} & $\begin{array}{c}\text { Вариационный ряд по } \\
\text { Ш }_{\text {вп }}\end{array}$ & \multicolumn{2}{c|}{$\begin{array}{c}\text { Ва риационный ряд } \\
\text { по Ш }\end{array}$} \\
\cline { 2 - 9 } & $\begin{array}{c}\text { Границы } \\
\text { классовых } \\
\text { интервалов, } \\
\text { мм }\end{array}$ & $\begin{array}{c}\text { Част. } \\
\text { вар. в } \\
\text { кажд. } \\
\text { классе }\end{array}$ & $\begin{array}{c}\text { Границы } \\
\text { классов. } \\
\text { интерв., мм }\end{array}$ & $\begin{array}{c}\text { Част. } \\
\text { вар. в } \\
\text { кажд. } \\
\text { классе }\end{array}$ & $\begin{array}{c}\text { Границы } \\
\text { классов. } \\
\text { интерв., мм }\end{array}$ & $\begin{array}{c}\text { Част. } \\
\text { вар. в } \\
\text { кажд. } \\
\text { классе }\end{array}$ & $\begin{array}{c}\text { Границы } \\
\text { классов. } \\
\text { интерв., } \\
\text { мм }\end{array}$ & $\begin{array}{c}\text { Част. } \\
\text { вар. в } \\
\text { кажд. } \\
\text { классе }\end{array}$ \\
\hline 1 & $206,5-211,4$ & 14 & $79,5-82,4$ & 7 & $82,5-85,4$ & 15 & $41,5-44,4$ & 8 \\
\hline 2 & $211,5-216,4$ & 24 & $82,5-85,4$ & 33 & $85,5-88,4$ & 31 & $44,5-47,4$ & 9 \\
\hline 3 & $216,5-221,4$ & 28 & $85,5-88,4$ & 48 & $88,5-91,4$ & 45 & $47,5-50,4$ & 16 \\
\hline 4 & $221,5-226,4$ & 67 & $88,5-91,4$ & 73 & $91,5-94,4$ & 62 & $50,5-53,4$ & 24 \\
\hline 5 & $226,5-231,4$ & 47 & $91,5-94,4$ & 41 & $94,5-97,4$ & 49 & $53,5-56,4$ & 43 \\
\hline 6 & $231,5-236,4$ & 32 & $94,5-97,4$ & 29 & $97,5-100,4$ & 31 & $56,5-59,4$ & 54 \\
\hline 7 & $236,5-241,4$ & 21 & $97,5-100,4$ & 12 & $100,5-103,4$ & 10 & $59,5-62,4$ & 42 \\
\hline 8 & $241,5-246,4$ & 12 & $100,5-103,4$ & 4 & $103,5-106,4$ & 5 & $62,5-65,4$ & 36 \\
\hline 9 & $246,5-251,4$ & 5 & $103,5-106,4$ & 3 & $106,5-109,4$ & 2 & $65,5-68,4$ & 12 \\
\hline
\end{tabular}

В результате обработки антропометрических исследований были получены диаграммы распределения размерных параметров, которые представлены в рисунке 1.

Распределение частот в вариационном ряду, как это видно по диаграмме распределения, таково, что наибольшее число одинаковых вариантов приходится на класс, который расположен по середине ряда. Вправо и влево от этого класса на диаграмме распределения число одинаковых вариантов в каждом классе постепенно убывает. Наименьшая частота вариантов наблюдается в первом и последнем классах. Такая закономерность в вариабельности значений признака в вариационном ряду наблюдается у всех антропометрических признаков.

Разработаем в среде Delphi Architect алгоритмы и программу для анализа статистических данных и поиска оптимальных значений коэффициентов нормального распределения:

$$
f(x)=\frac{1}{\sigma \sqrt{2 \pi}} e^{-\frac{(x-\mu)^{2}}{2 \delta^{2}}} .
$$

После несложных преобразований выражения (1) и замены, получим более удобную формулу для нормального распределения:

$$
f(x, a, b, c)=a e^{-\frac{(x-c)^{2}}{2 b}}
$$

Для каждого из 4-х распределений (рис.1) зададим начальные значения коэффициентов. Оптимальные параметры будем искать в окрестности начальных значений. 


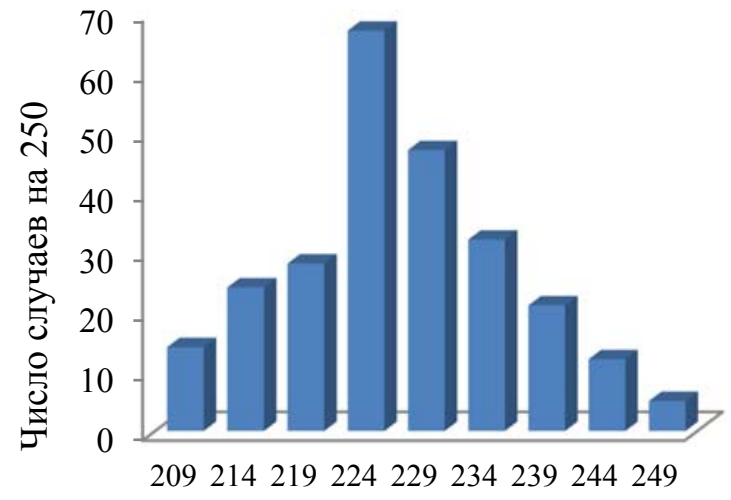

Длина стопы, мм

a

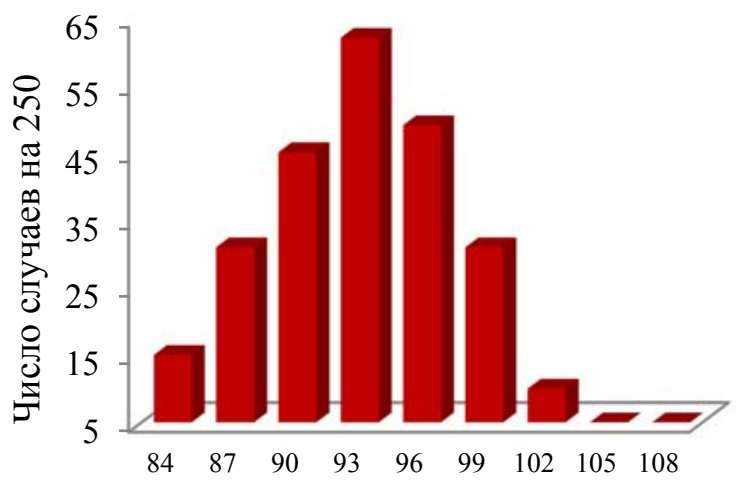

Ширина внутреннего пучка, мм

B

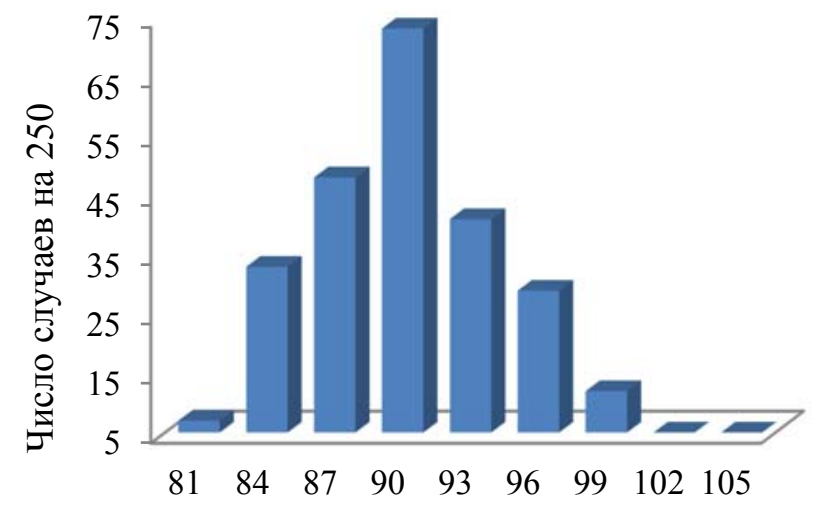

Ширина наружного пучка, мм

б

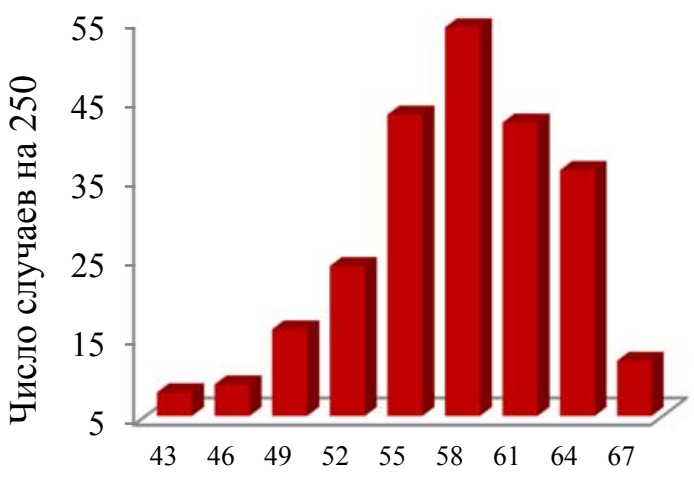

Ширина пятки, мм $\Gamma$

Рисунок 1 - Распределение стопы по размерным признакам:

a - длина стопы, б - ширина наружного пучка, в - ширина внутреннего пучка, г - ширина пятки.

\section{unit Unit1;}

interface

uses

Winapi.Windows, Winapi.Messages, System.SysUtils, System.Variants, System.Classes, Vcl.Graphics,

Vcl.Controls, Vcl.Forms, Vcl.Dialogs, Vcl.StdCtrls, VCLTee.Series,

VCLTee.TeEngine, VCLTee.TeeProcs, VCLTee.Chart, Vcl.ExtCtrls, Vcl.ComCtrls;

type

TForm $1=$ class $($ TForm $)$

Panel1: TPanel;

Chart1: TChart;

Series 1: TLineSeries;

Series2: TBarSeries;

Button1: TButton; 


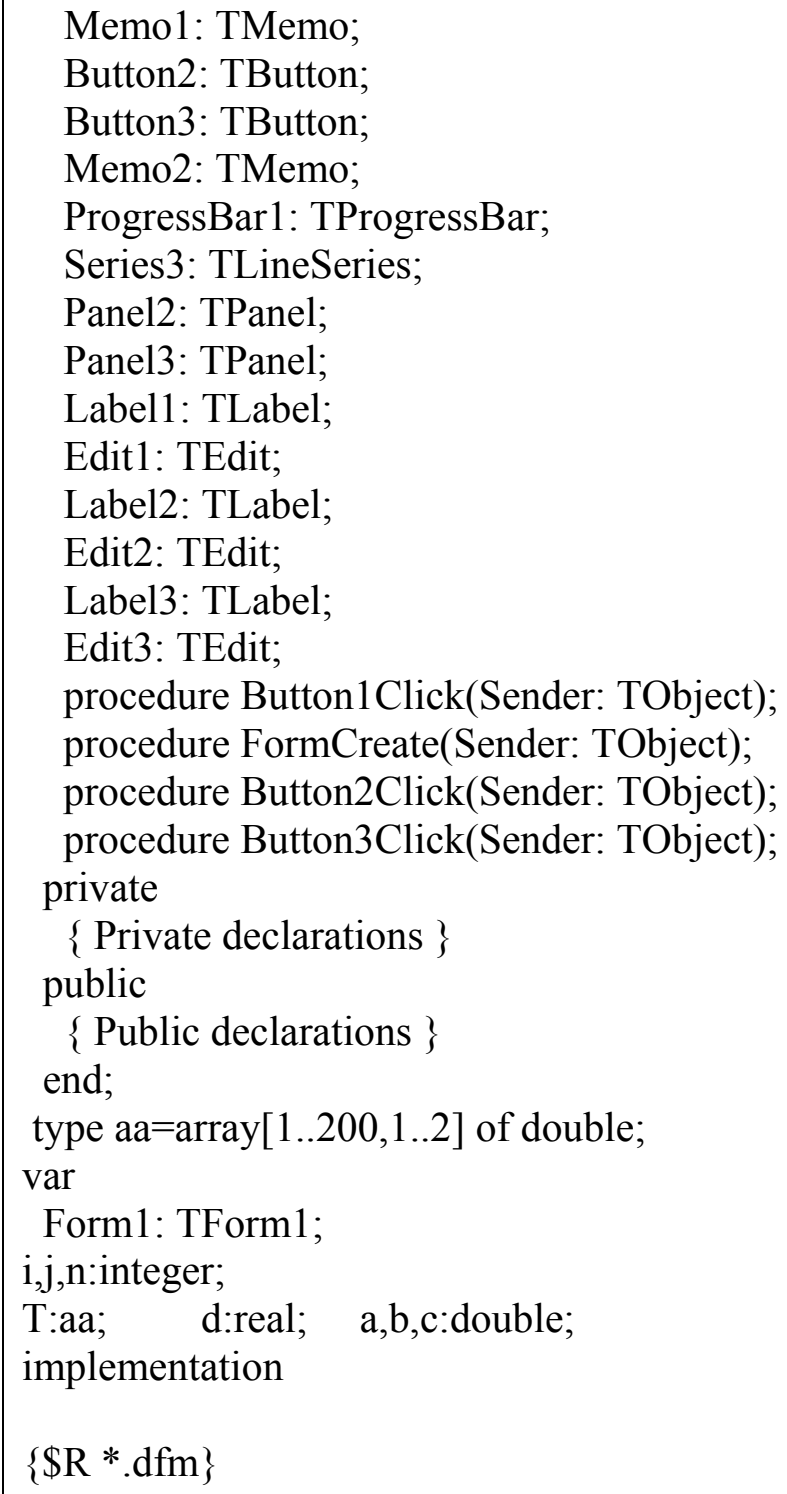




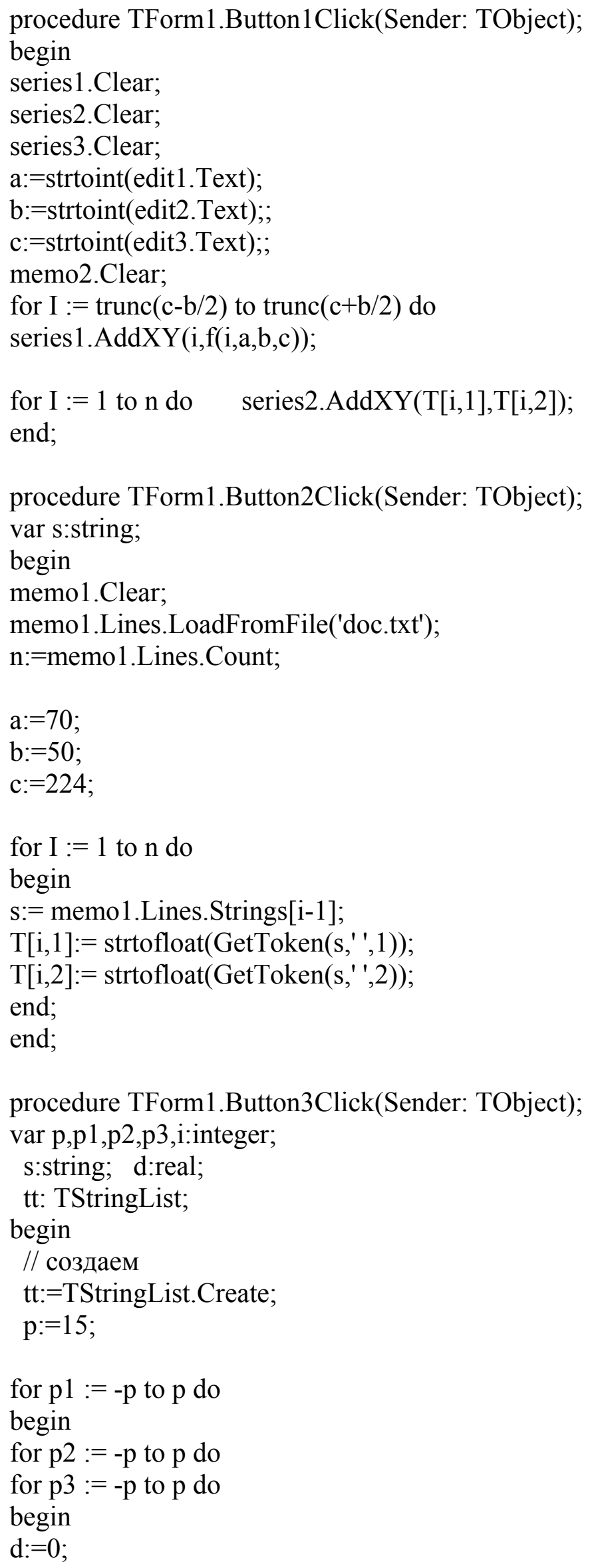




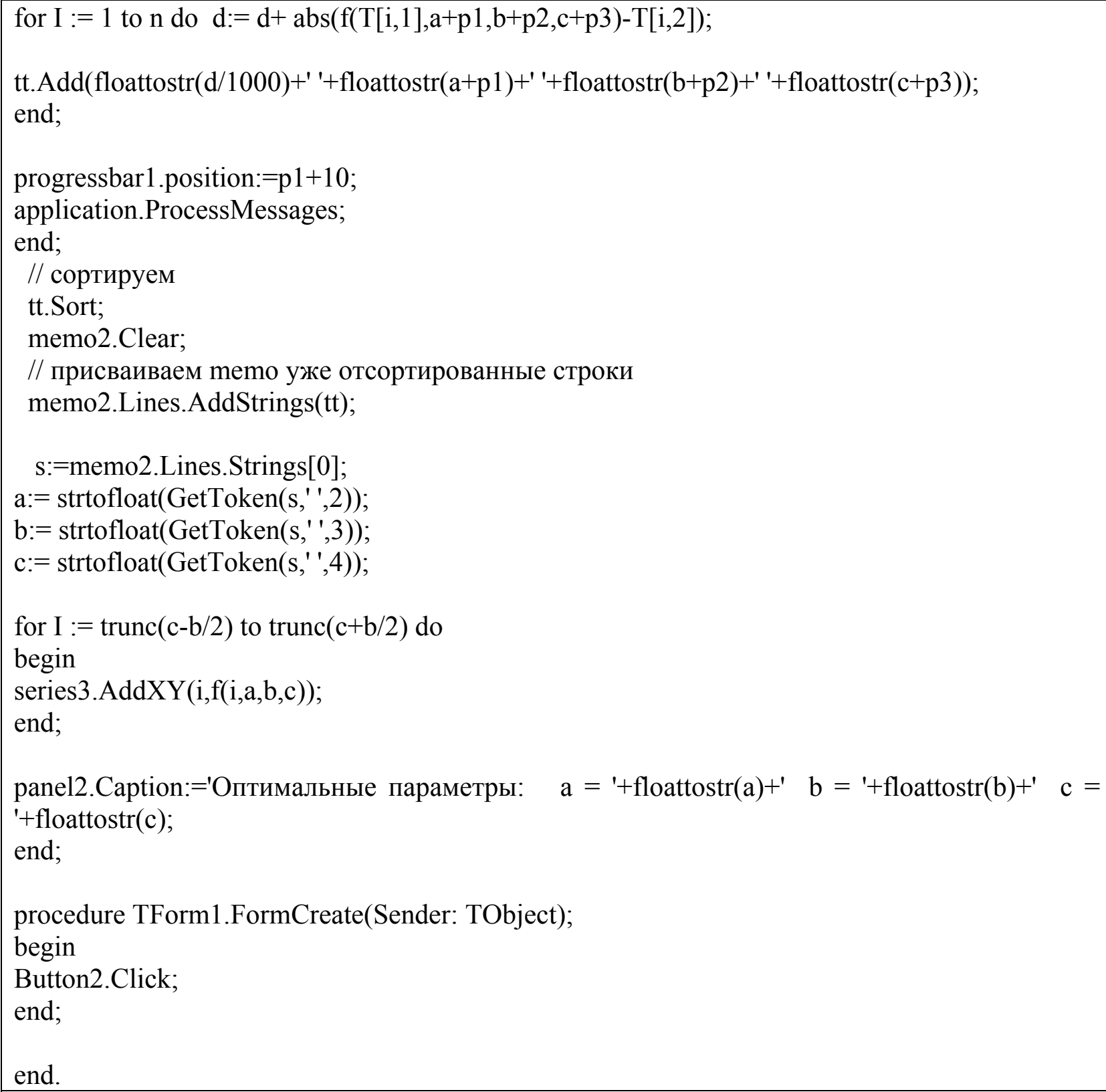

В результате проведенного анализа получим следующие оптимальные нормальные распределения соответственно для четырех диаграмм:

Таблица 2

Оптимальное нормальное распределение.

\begin{tabular}{|l|l|c|}
\hline$№$ & \multicolumn{1}{|c|}{ Описание } & Оптимальное нормальное распределение \\
\hline 1 & Длина стопы (рис.1, а) & $f(x)=57 \cdot e^{-\frac{(x-225)^{2}}{140}}$ \\
\hline 2 & Ширина наружного пучка (рис.1, б) & $f(x)=58 \cdot e^{-\frac{(x-90)^{2}}{52}}$ \\
\hline 3 & Ширина внутреннего пучка (рис.1, в) & $f(x)=57 \cdot e^{-\frac{(x-93)^{2}}{60}}$ \\
\hline 4 & Ширина пятки (рис.1, г) & $f(x)=49 \cdot e^{-\frac{(x-58)^{2}}{72}}$ \\
\hline
\end{tabular}




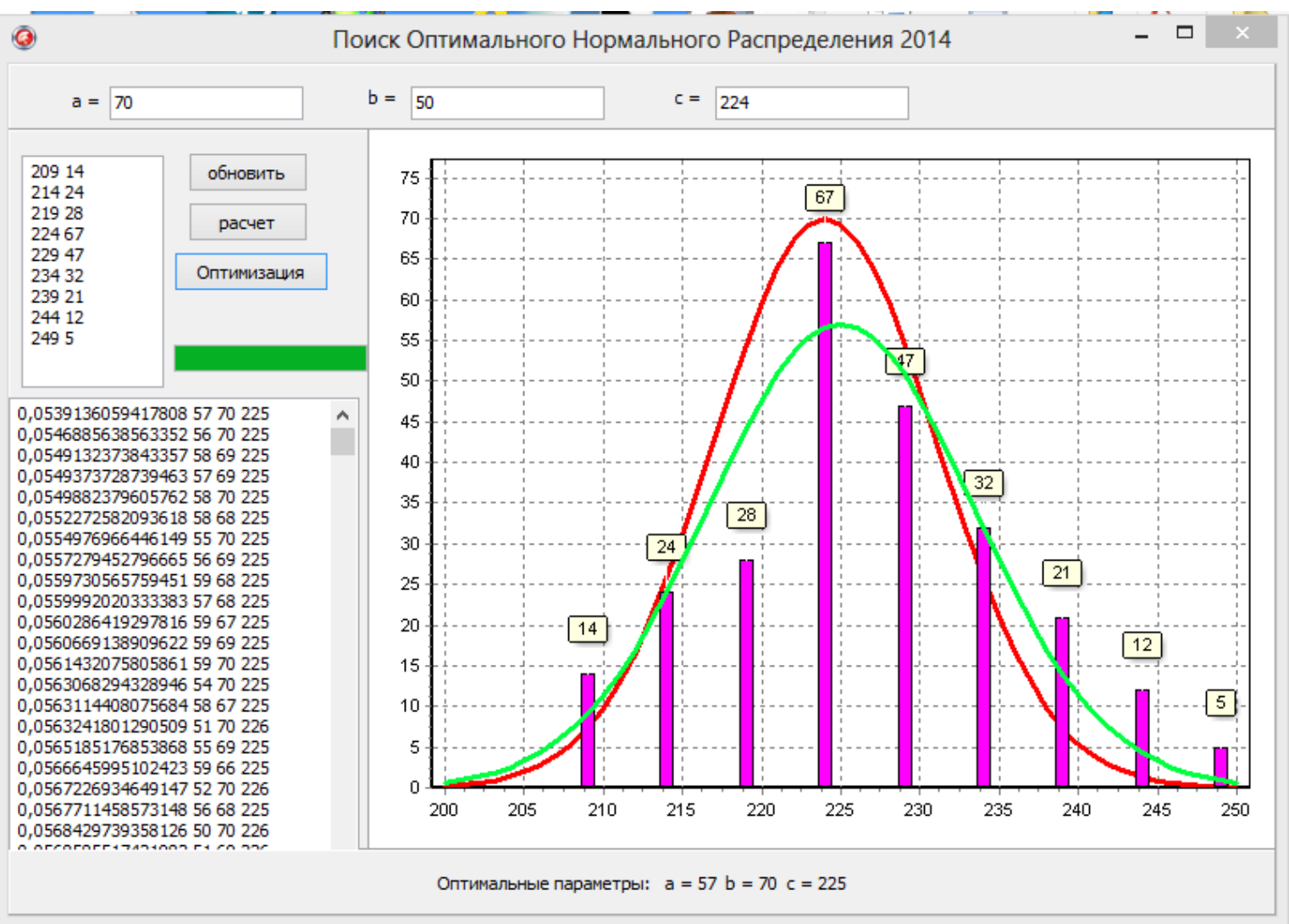

Рисунок 2 - Оптимальное нормальное распределение. Длина стопы (рис.1, a).

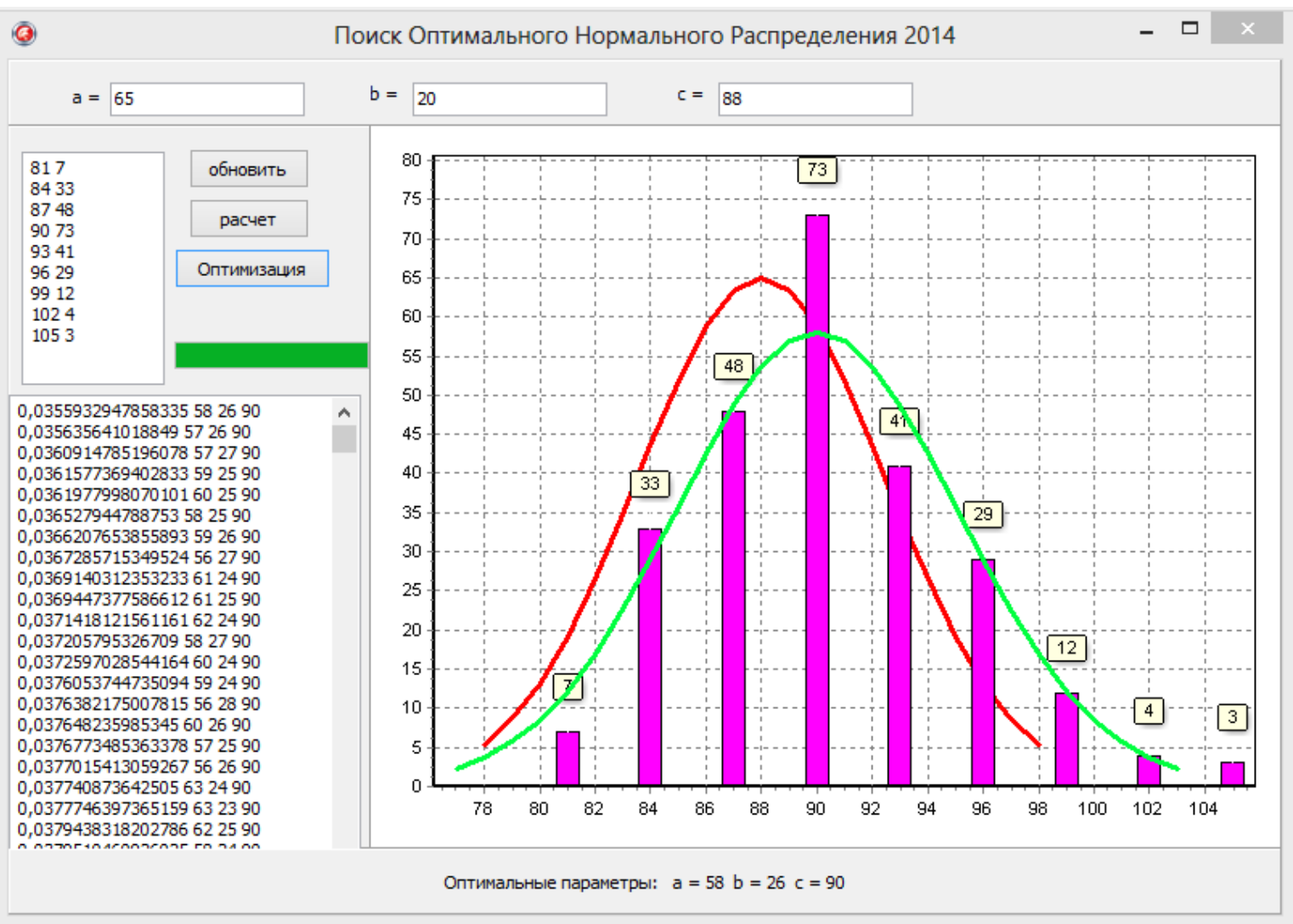

Рисунок 3 - Оптимальное нормальное распределение. Ширина наружного пучка (рис.1, б). 


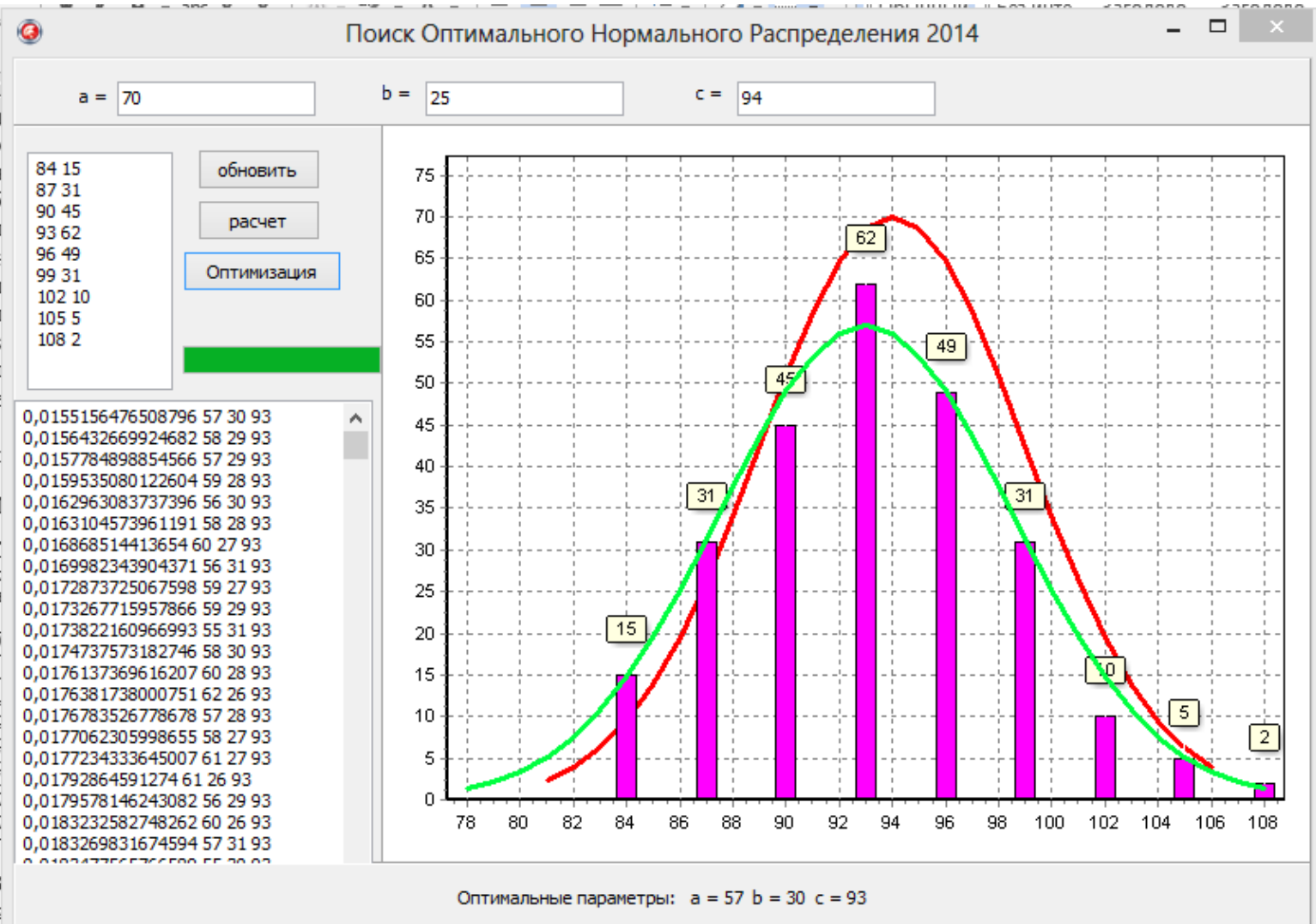

Рисунок 4 - Оптимальное нормальное распределение. Ширина внутреннего пучка (рис.1, в).

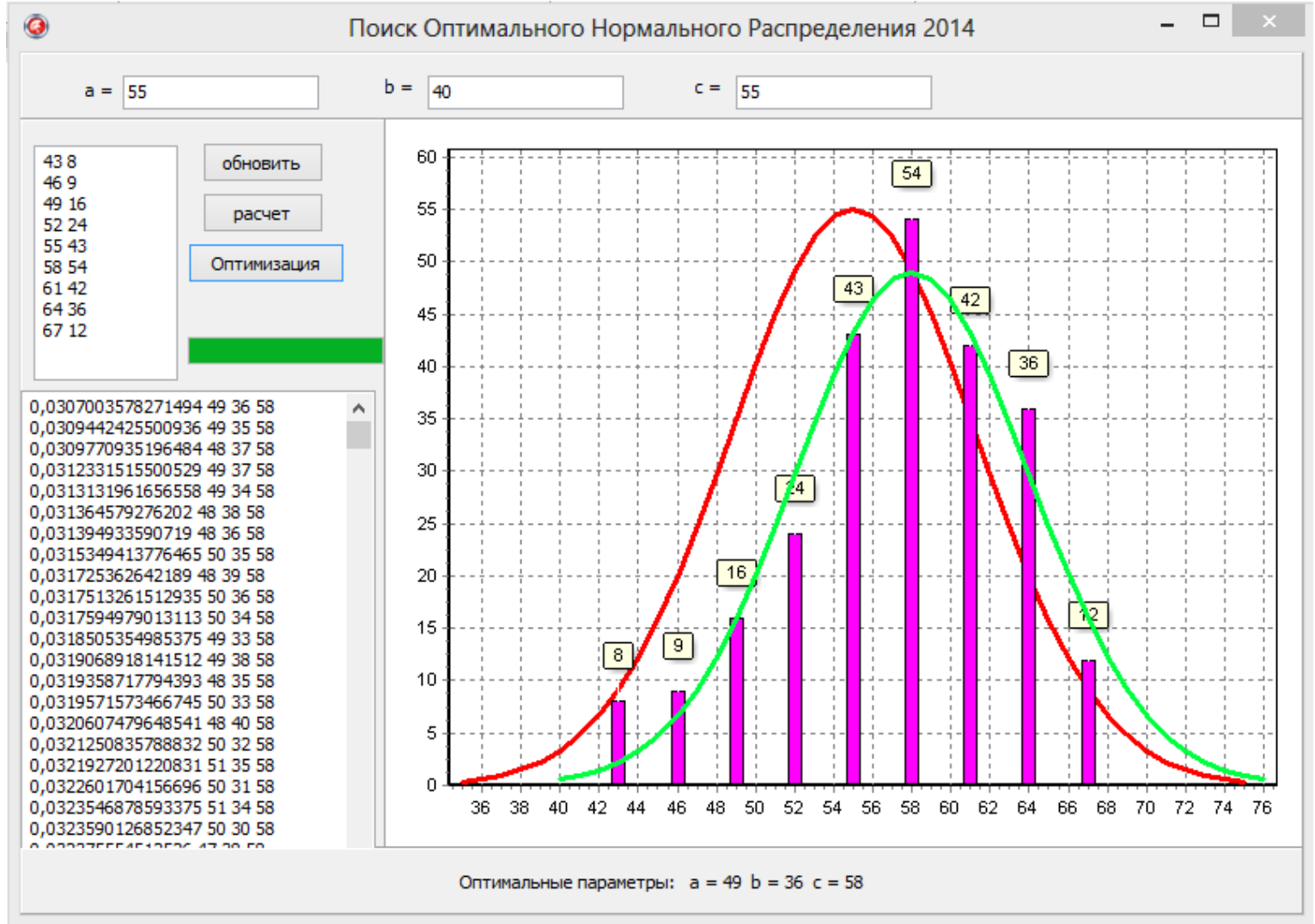

Рисунок 5 - Оптимальное нормальное распределение. Ширина пятки (рис.1, г). 


\section{ВЫВОДЫ}

По представленным диаграммам видно, что распределение стоп по основным размерным признакам близки к нормальному распределению.

Получены оптимальные аналитические формулы нормального распределения для расчета исследуемых признаков. Результаты расчета основных коэффициентов нормальных распределений исследуемых размерных признаков представлены в таблице 2 и на рисунках 2-5.

\section{References:}

1. Shevtsov AN, Abzalbekov B, Munasipov SE (2013) CONSTRUCTION OF MATHEMATICAL MODELS OF ORTHOPEDIC INSOLES FOR SHOES PATIENTS WITH DIABETES MELLITUS. ISJ Theoretical \& Applied Science 5 (1): pp.113-120. doi: http://dx.doi.org/10.15863/TAS.2013.05.1.19

2. Abzalbekuly B, Munasipov SE, Onlabekova A, Togguzbaev K (2013) Anthropometric research of feet of population in the republic of Kazakhstan. Trudy mezhdunarodnoy nauchnoy e-konferentsii «Seattle-2013: 4th International Academic Research Conference on Business, Education, Nature and Technology». g.Siettl. USA. 4-5 noyabrya 2013.

3. Grdzelidze M, Katamadze A, Shalamberidze M (2008) O spetsifike trebovaniy, pred"yavlyaemykh k obuvi s uchetom patologii stopy. Zhurnal Georgian Engineering News. No.4, pp. $199-201$.

4. Grdzelidze MG, Katamadze AG, Shalamberidze MM (2011) Formirovanie trebovaniy, pred"yavlyaemykh k diaobuvi po kategoriyam bol'nykh sindromom «Diabeticheskaya stopa». VISNIK KNUTD. No.5, pp. 125-128.

5. Katamadze A, Grdzelidze M, Shalamberidze M (2009) Diabaticheskaya stopa i pred"vlyaemye trebovaniya $\mathrm{k}$ diaobuvi. International scientific journal -"Georgian scientific news". No.4, pp. 11-14.

6. Udovichenko OV, i dr. (2006) Rekomendatsii po izgotovlenyu ortopedicheskoy obuvi dlya patsientov s sakharnym diabetom. Sakharnyy diabet. No.3, pp. 46-50. https://ru.wikipedia.org/wiki/\%CD\%EE\%F0\%EC\%E0\%EB\%FC\%ED\%EE\%E5 \%F0\%E0\% F1\%EF\%F0\%E5\%E4\%E5\%EB\%E5\%ED\%E8\%E5 Accessed: 2014 Sep 20.

8. Abzalbekuly B, Binazarov SZh, Munasipov SE (2012) K probleme sozdaniya ratsional'nykh konstruktsii diaobuvi. Materialy respublikanskoy nauchno-prakticheskoy konferentsiy magistrantov, doktorantov i molodykh prepodavateley: «Nauka i sovremennost'-2012», Taraz.

9. Abzalbekuly B, Munasipov SE, Yankauskayte V, Toguzbaev KU (2013) Vliyanie konstruktsii i materialov ortopedicheskikh izdeliy na komfortnye svoystva obuvi. «VESTNIK» ATU. No.2, pp. 20-24.

10. Cover Thomas M (2006) Elements of Information Theory. John Wiley and Sons, 254.

11. (2009) «Maximum Entropy Autoregressive Conditional Heteroskedasticity Model». Journal of Econometrics (Elsevier): 219-230. 